\title{
Infrasound in the Atmosphere of the Earth
}

\section{Galyna Ivanovna Sokol}

Faculty of Physics and Technology, Dnepropetrovsk National University Named Of Oles Gonchar, Dnepropetrovsk, Ukraine

Email address:

gsokol@ukr.net

To cite this article:

Galyna Ivanovna Sokol. Infrasound in the Atmosphere of the Earth. European Journal of Biophysics. Vol. 3, No. 1, 2015, pp. 1-4. doi: $10.11648 /$ j.ejb.20150301.11

\begin{abstract}
Infrasonic as the factor of the influence on the climate of the Earth is submitted. The results of researches between the infrasonic and the sunny activity are showed. It is necessary the ecological aspects in intercommunications between the infrasonic and atmosphere effects, parts of biosphere to identify.
\end{abstract}

Keywords: Infrasonic, Fluctuations, Space Weather, Climate, Biosphere of the Earth

\section{Introduction}

In this work it is makes the analysis: in what case we have the infrasound in atmosphere. We have some factors, that infrasound waves are produced. Specific influence of low frequency acoustic waves on living beings are reviewed. The theme is based on the fact that the resonance frequencies of the most important human organs are in the frequency range 0.5-20 Hz (L. Pimonov, V. Gavro, E.N. Malyshev, M.A. Isakovich, A.V. Rimsky - Korsakov, V. Tempest). Infrasound signal attenuation in atmosphere is not enough, which speaks proportionality of a decay factor to a frequency quadrate. Therefore sometimes an infrasound term as "an ultrasonic neutrino». Uptake of energy of infrasonic waves by frequency in the inferior atmospheric slices compounds of $0,1 \mathrm{~Hz} 2 \cdot 10-9 \mathrm{~dB} / \mathrm{km}$. Therefore, the study of infrasound is very important.

\section{Main Part}

Formation of space and atmospheric weather, changes in the Earth's climate is now involved in many world organizations. In Ukraine it is States Space Agency and anthers organization that are the party of it from Dniepropetrovsk, Kiev, Lvov. In world that are Intergovernmental Oceanographic Commission, World Metrological Organization. In USA it is National Center for Atmospheric Research, Colorado, Boulder; University Corporation for Atmospheric Research. Intergovernmental Group of Experts on Climate Change (IPCC). It is agreed with the National Academy of Sciences of the "Group of eight, G8 an international club that unites government
(United Kingdom, Germany, Italy, Japan, Russia, USA, France and Japan) and others. Intergovernmental Panel on Climate Change, IPCC) it is a big club that unites government United Kingdom, Germany, Italy, Japan, Russia, USA, France and Japan) and others. Developed a research program on climate change of the Earth. For example, World Weather Research Program, World Climate Research Program, International Geosphere Biosphere Program.

Recently more and more attention it is given to every possible oscillating motions of atmosphere, including to infrasonic waves. The purpose of the present operation is examination of generation of infrasonic waves in an Earth's atmosphere and their interaction with the atmospheric phenomena. At the present stage less all is clarified atmospheric links at levels below $200 \mathrm{~km}$. Without their definitive installation it will be difficult to solve completely and bottoms of is solar-terrestrial relationships of cause and effect.

Infrasonic oscillations in an Earth's atmosphere grow out of activity of the numerous parents: earthquakes (earth crust oscillations), a tsunami. It is known that the infrasound sources are: eruptions of volcanoes, falls, thunder-storms, oscillations of a surface of the sea, forest fires, the strong wind, turbulence of atmosphere, activity of the person (explosions, gun shots, gyration of lobes wind current generator, transport actuators), electromagnetic radiations, motions of meteors, galactic rays, gravitational actions of moon and the Sun, corpuscular flows from the Sun. It is earthquake, mountains, the storms of the sea, the sources of vortices in atmosphere, the sun activity and geomagnetic variations and others [1].

The scientists of the department of Ukrainian Institute of 
Space of State Space Agency of Ukraine Science Academy (SSAU) in Lvov registered the infrasound of earthquakes in Turkey (A.A. Negoda, S.A. Soroka and other scientists) [2]. They defined that the infrasound is connected with the sun activity. When the sun activity is high the infrasound decries in atmosphere and vice versa when the sun activity is decries high in atmosphere the level of the infrasound is drops. Now we have others questions like: how does infrasound connect with sun's activity? Analysis of seismicity phenomenon with an infrasound that was made in Turkey on a continuance 19972000 has been carried out. Spectral characteristics of an infrasound and seismic activity will well be agreed. On fig. 1 spectroscopic densities of diurnal energy of an infrasound and seismic activity for a continuance 1997-2000 are displayed, that infrasonic oscillations "are sensitive" to changes of seismic activity in radius to $2000 \mathrm{~km}$. The optimum dimension of radius of this field lies within the limits $1000-1500 \mathrm{~km}$. The greatest interest to IS - waves appear after the represents analysis of the phenomenon's in IOA that was made before the catastrophic earthquakes in region.



Fig. 1. Spectroscopic densities and a coefficient of seismic activity during 1997-2000.

An examination of infrasonic spectrums in an Earth's atmosphere before large earthquakes has displayed their

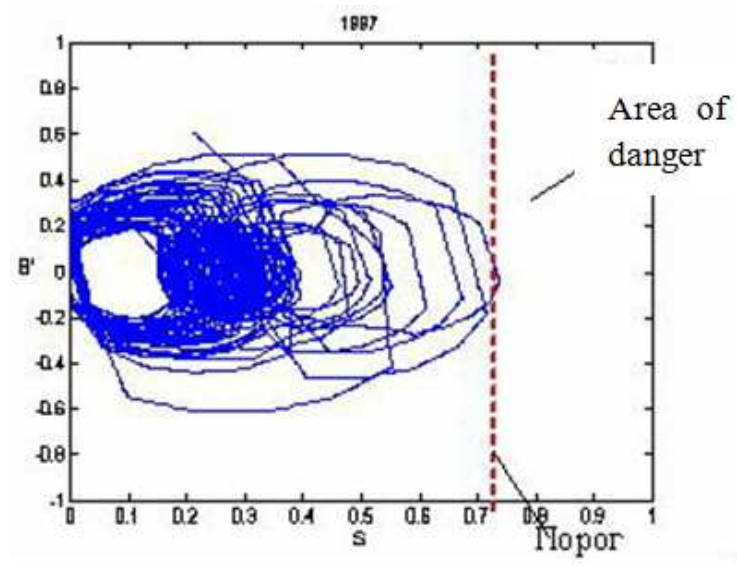

characteristic changes before large earthquakes. Infrasonic and gravity waves above $100 \mathrm{~km}$ where their voltage is great, continuously raising and sinking various atmospheric slices, in addition promote intermixing of various components. On fig. 2. energy of an infrasound and solar activity in 19972000 have displayed.

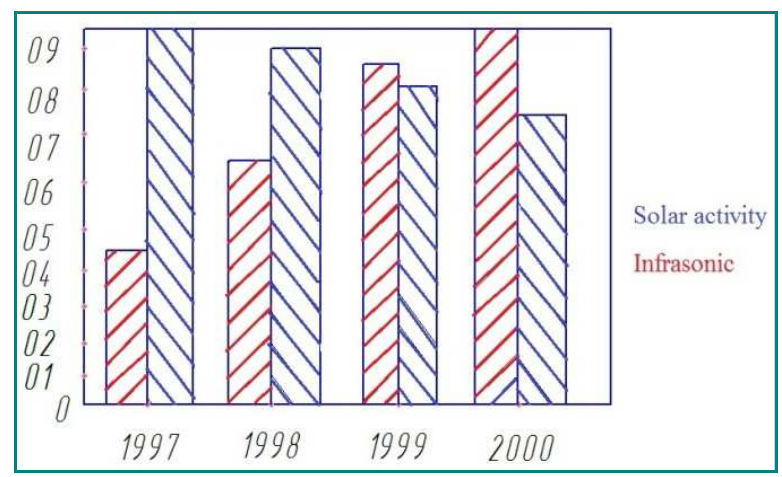

Fig. 2. Energy of an infrasound and solar activity in 1997-2000.

On fig. 3 phase portraits of seismic activity for a continuance 1997-2000 are displayed. On an abscissa axis it is put aside values of a coefficient of seismic activity (in this case the normalized values of a quadrate of the maximum magnitude in the given region). On an axis of ordinates the normalized derivative from the function constructed on values of a coefficient of seismic activity is put aside. Apparently from fig. 3 phase trajectories are drawn to unconstructive field. Only at catastrophic earthquakes the phase trajectory goes out attraction field. And, the exit of a phase trajectory from field of an attraction and an inlet in dangerous field (in drawing it is scored by a vertical dotted line) does not descend instantaneous. It descends for some days.

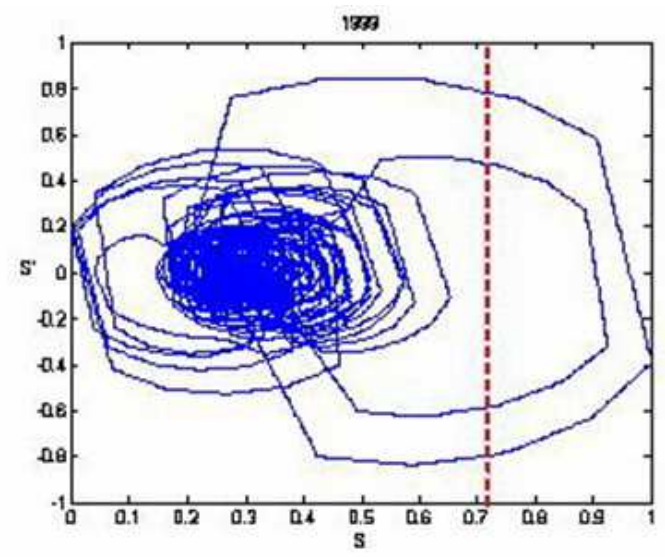

Fig. 3. Phase portraits of seismic activity during 1997-2000

The scientist V.I. Krasovsky [3] proposed to divide the atmosphere to lower and upper atmosphere. In lower atmosphere we have the clouds, that give us the rain or we have the precipitates. Earthquakes generate infrasound in the atmosphere. Infrasound reaches the surface of storm clouds, affects the surface. Rain intensifies. So infrasound manifests itself as an integral part, which forms the climate and the impact on the biosphere.

But in upper atmosphere the astronauts detected the silvery clouds (fig. 4). Various space particles like a little meteorites travel from the space and stop and crystallizes at the distance of $80 \mathrm{~km}$ from the surface of the Earth. And turn into the 
silvery clouds. At first astronauts noticed the wave structure of these clouds. And the length of the waves quells to the length of infrasonic waves. These clouds never produce precipitates. They only produce the shade on the surface of Earth. It depends on temperature and pressure of air in atmosphere. It is factor of the influence of the climate of the Earth.

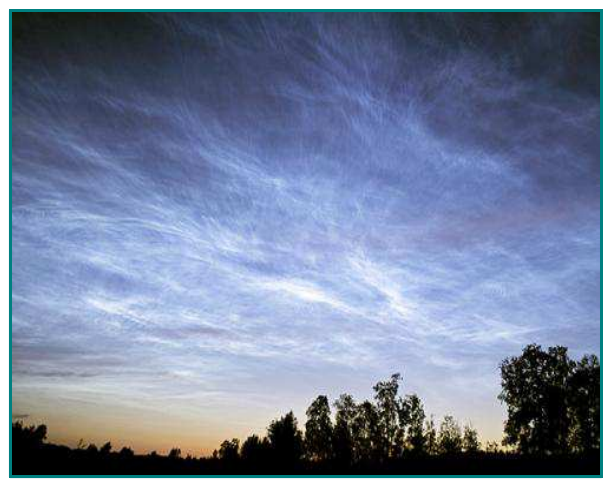

Fig. 4. The silvery clouds.

The wave structure with the length of infrasound is because earthquakes produce the infrasound. The infrasonic waves of the quakes travel in high atmosphere. They amplitude not change a lot. But on high $80 \mathrm{~km}$ there are infrasonic waves. There is in sound channel and infrasonic waves (IS - waves) turn on 90 degrees and continued to travel in direction that is parallels on surface on the Earth. The infrasonic waves moved by meridians to poles of the Earth.

When infrasonic waves connecting with magnetic particles we can see the phenomenon of aurora at North Pole [3]. Because geomagnetic particles connected with infrasonic waves. The conducted operations have displayed, that this interaction makes essential impact on an ionosphere. It is proved, that perturbations of an ionosphere of an infrasound from earthquakes and eruptions of volcanoes it is accompanied by a birth of magnetic storms. Earlier the scientists were supposed and considered that the disturbing in an ionosphere (IOA) is connect only with solar flares. At the present stage the scientists are connect the disturbing in an ionosphere with IS - waves.

The factor, making the significant impact on infrasonic oscillations of atmosphere, is seismic activity. And it can be an exposure on preparatory processes and the link of intensity of seismic processes. It can be connect with solar activity. The scientists found effect at analysis of global seismicity and 11-year-old solar cycles. Influence of seismic activity on IOA is very complex process and is not reduced only to piston radiance fluctuating lithosphere plates. Here it is necessary to consider manifold physicochemical processes, both in lithosphere, and in atmosphere. IOA can be generated by gaseous release from flaws at the lithosphere increase of seismic activity, oscillations lithosphere plates, aerosol in homogeneities in atmosphere. IOA can create on a surface of the Earth alternate stresses and in pour on the significant depths in lithosphere. Infrasonic oscillations influence on the velocity of fluids travel, on the electrical fields and on local seismic oscillations by stimulation in the lithosphere. Thus, the infrasound in atmosphere can be generating as effect of seismic oscillations and awake the influence on the atmosphere. In character of interchange by vibration energy between lithosphere and atmosphere processes of preparation of large earthquakes can show. For examinations of the infrasonic canal of lithosphere-atmospheric links it has been introduced two coefficients of seismic activity. The first proportional to a quadrate of the maximum magnitude in the given day in the given region, second - to a quadrate of the total of magnitudes of all seismic events with magnitude $\geq 3$ for a day in the given region. It was considered two regions. One of it is dimensioned on a longitude $10^{\circ}-45^{\circ} \mathrm{E}$ and latitude $35^{\circ}-60^{\circ} \mathrm{N}$, and second - on a longitude $10^{\circ}-55^{\circ} \mathrm{E}$ and latitude $20^{\circ}-60^{\circ} \mathrm{N}$. The first and second regions powered up the basic bands of heightened seismicity of the central and east Europe, and also Turkey. Infrasound's measuring was made in a point with co-ordinates $48^{\circ} 41^{\prime} \mathrm{N}, 26^{\circ} 30^{\prime} \mathrm{E}$ [2].

It is noted, that the greatest level the infrasonic background reaches during the maximum heating of atmosphere. One of the parents of this phenomenon is forest fires. For last two years the reality of such processes is confirmed. For a long time already the radiant of warming up of the upper atmosphere represents all views of infrasonic oscillations, powering up and interior gravity waves. Most awake investigated died these phenomena Canadian geophysics Haynes. Scientists executed necessary measuring with the help infrasound metric complex which powers up two modules of pressure. Modules have disposed apart 85 meters from each other. Measuring conducted within 5 minutes, then 5 minutes a rest and again measuring. Having analyzed the gained effects, they have come to conclusion, that level of an infrasonic background is not constant. It varies both throughout a year and within days. In the afternoon it strengthens, reaching peak about 11 hours per winter time and approximately at 16 o'clock in the summer. That is the greatest level the infrasonic background reaches during the maximum warm-up of atmosphere.

Therefore reaching of stratums of an ionosphere by the infrasonic waves generated in an Earth's atmosphere by operation vertically-axial wind aggregate is real.

From the above-stated follows, that to a variation of frame of the upper atmosphere, geomagnetic disturbances and auroras does not give in to explanation only agents of a solar parentage. Inferior atmosphere is essential modulates effects of a solar parentage. One of the parameters characterizing auroras, the ionic density is. In an aurorally region the nonuniform ionization arises because of non-uniformity of interfering flows of vigorous charged particles. It appears that the ionic density fluctuates because of gravitational and infrasonic waves in which there are density changes of atmosphere and altitudes of levels of its identical values. The fluctuation field is usually termed as diffuse stratum F. The USA in 1969, the USSR in 1973 and the USSR together with France in 1975 have made experiments on making of artificial auroras during which time from a missile at the altitude in some honeycombs of kilometers the bundle of electrons of high energies was injected in atmosphere. 
Realization of controllable experiments together with tetramerous observations uncloses new trajectories to examination of auroras and processes in the upper atmosphere.

In a band of auroras there are jet currents. Jet currents in a band of auroras are rather impulsive and consequently; oscillations of a manifold spectrum of infrasonic waves with continuances from seconds till several o'clock also can lead. The more wind speed, more effective an energy conversion of a jet current in infrasonic waves. Interaction of electromagnetic radiation with optical in homogeneities of atmosphere can lead to generation of ultrasonic oscillations in a broad band of frequencies. It is necessary to expect therefore, that in a spectrum of infrasonic oscillations of atmosphere rhythmic of solar activity should show. As a result of experiments on observation of electromagnetic responses to infrasound perturbations in the atmosphere, created by means of a portable ultrasonic emitter, link of an infrasound with geomagnetic variations is proved. Thus, the Sun, interplanetary medium, atmosphere and lithosphere and represent unified system, and the essential role in processes of their interaction is played by infrasonic waves. The infrasonic and magneto hydrodynamic waves originating both in the uppermost atmosphere, and behind its limits below and in a magnetosphere. Recently a lot of attention is given to the infrasonic and magneto hydrodynamic waves originating both in the uppermost atmosphere, and behind its limits below and in a magnetosphere. Pressure of sound waves invokes atmosphere inflating. Waves with the frequency exceeding $0,1 \mathrm{glc}$, are customary waves and are spread with velocity of a sound ( $g$ - acceleration of gravity). Waves with frequency, smaller $0,1 \mathrm{glc}$, go with a little bit smaller velocity. Last, named by gravity waves, always have a wave length exceeding altitude of a homogeneous atmosphere. Thus, the wave length of gravity waves will be not less than hundred kilometers.

Thereof in various bands of atmosphere temperature lapse rates and the thermal instabilities generating IOA are organized. The organized infrasound can influence fluctuations of intensity of interaction of ultra rays with atmospheric aerosols. In earth crust percussions and vibrations of very low sound frequencies from the diversified radiant are, including from explosions are observed. For an infrasound small uptake in various mediums owing to what infrasonic waves in air, water is characteristic and in earth crust can be spread to very far distances.

The wind power concerns to one of perspective directions of the solution of the given problem. Wind energy installations are typical emitters of an infrasound. Major diffusion to the world curls to Darya and Savonius vertically-axial (VA) phylum [4] have gained two-and three-blade is horizontal-axial (HA) wind energy installations (WEI) fan phylum, and also. At periodic action on medium of gyrating lobes the sound field is generated. The circular rotation frequency of three-blade curls ВЭУ-250С and ВЭУ-500C of Ukraine compounds 47,6 rpm.
Their three-blade curls generate in a surrounding medium an infrasound with frequency of $2,4 \mathrm{~Hz}$. The circular rotation frequency of two-blade curls IN WEG-0020 and WEG-0030, developed by the International Is scientific-industrial corporation "VESTA", compounds 28-90 rpm. Their two-blade curls generate in a surrounding medium an infrasound with frequency of 2-7 Hz. It is displayed, that for the lobe in length of $12 \mathrm{~m}$ at velocities of an airflow, smaller $10 \mathrm{~km} / \mathrm{s}$, the frequency of a fundamental component calculated on a Stokes formula, is infrasonic and abandons $0,4 \mathrm{~Hz}$. Performances of a sound field of a curl are horizontal-axial wind electric power stations are calculated on a procedure developed in operations [4]. Here it is displayed, that the acoustic field propeller has a directional characteristic. As a result of the conducted examinations it is erected, that the noise level in an acoustic field propeller on a very low sound frequency apart $300 \mathrm{~m}$ from wind aggregate compounds of $2,4 \mathrm{~Hz} 63 \mathrm{~dB}$.

\section{Summary}

Infrasound radiant's in an Earth's atmosphere are systematized. Link of an infrasound with the phenomena in upper and in inferior atmospheres is viewed. Changes in the infrasound spectrum, invoked lithosphere are featured by processes. An Energy of an infrasound increases at solar activity slope. 5-10 days prior to large earthquakes the spectrum and the phase glow iris of infrasonic oscillations in atmosphere that can become a bottom for making of a new method of the forecast of earthquakes is essential variants. The infrasound from earthquakes and fires can serve as a harbinger, a signal and the parent of cataclysms (explosions of methane ice). Analysis of a frequency characteristic of an acoustic field has displayed, that by operation vertically-axial wind aggregates in an Earth's atmosphere infrasonic waves by frequency of 4-7 Hz are generated. Infrasound presence in an Earth's atmosphere at growing powers vertically-axial wind aggregates guesses realization of the further analysis of interaction of infrasonic acoustic fields with sunlight.

\section{References}

[1] Сокол Г.И. Особенности инфразвуковых процессов в инфразвуковом диапазоне частот. - Днепропетровск: Промінь, 2000. - 136 р.

[2] Негода А.А., Сорока С.А. Акустический канал космического влияния на биосферу Земли // Космічна наука і технологія. - 2001. - Т.16, № 5/6. - р. 85-93.

[3] Красовский В.И. Штили и штормы в верхней атмосфере М.: Наука, 1971. - 136 с.

[4] Сокол Г.И. Инфразвук - экологически вредный фактор в ветроэнергетике // Тр. Междунар. Акуст. симп.“Консонанс - 2005”: Киев, 27-29 сентября 2005: Сб. науч. Тр. - К., 2005. - p. 283 - 290. 